\title{
Review: evidence does not support the effectiveness of over the counter preparations for acute cough
}

\author{
Schroeder K, Fahey T. Over-the-counter medications for acute cough in children and adults in ambulatory settings. \\ Cochrane Database Syst Rev 2001;(4)CD001831 (latest version 25 May 2001).
QUESTION: In children and adults with acute cough, are over the counter (OTC) cough preparations effective for relieving symptoms?

\section{Data sources}

Studies were identified by searching 5 databases; scanning reference lists and personal files of references; and contacting authors and manufacturers.

\section{Study selection}

Studies were selected if they were randomised, placebo controlled studies (RCTs) of OTC preparations for acute cough in children and adults in outpatient settings. RCTs of chronic cough ( $>3$ wks duration), cough resulting from underlying respiratory disease, or cough induced artificially by inhalation of chemicals were excluded, as were studies of non-oral, herbal, or homeopathic preparations.

\section{Data extraction}

Data were extracted on participant characteristics, smoking status, study setting, randomisation, blinding, loss to follow up, definition of illness, drug dosage, frequency and duration of treatment, and outcomes (cough outcome such as severity, frequency, amount of sputum, and symptoms; and adverse effects).

\section{Main results}

21 RCTs ( $\mathrm{n}=2604)$ met the selection criteria. 15 RCTs involved adults $(\mathrm{n}=2166)$ and 6 involved children $(\mathrm{n}=438)$. Antitussives: in adults, 2 RCTs showed that codeine was not more effective than placebo for reducing cough in adults; 1 RCT found that dextromethorphan reduced coughing compared with placebo, whereas another found no difference. 1 RCT of moguisteine showed no difference in cough outcomes except in a subgroup of adults with more severe night cough. The moguisteine group had more side effects than the placebo group ( $22 \%$ $v 8 \%$, p value not reported). 1 RCT (57 children) showed that neither dextromethorphan nor codeine was more effective than placebo for reducing cough. Expectorants: 1 RCT of guaifenesin in adults showed greater self reported improvement in cough frequency and intensity $(75 \% v$ $31 \%, \mathrm{p}<0.01)$. The other RCT showed similar improve- ment in cough in both treatment and control groups, but guaifenesin led to more adults with reduced sputum thickness $(96 \%$ v 54\%, $\mathrm{p}=0.001)$. Mucolytics: in adults, 1 RCT of Bisolvon linctus showed less frequent coughing in the treatment group than in the placebo group $(8.6 \% \mathrm{v}$ $15 \%, \mathrm{p}<0.02)$. In children, 1 RCT of letosteine showed a mean difference of 0.2 in favour of the letosteine group on a 4 point symptom scale $(\mathrm{p}<0.01)$. Antihistamine decongestant combinations: in adults, 1 study of loratadine/ pseudoephedrine showed no difference in cough scores between groups, and another showed lower severity of cough in the dexbrompheniramine/pseudoephedrine group than in the placebo group (mean score $1.4 v 2.0$, $\mathrm{p}<0.05)$. In this study, the treatment group had increased dizziness and dry mouth $(\mathrm{p} \leqslant 0.01)$. In children, 2 RCTs showed that antihistamine decongestant combinations were not significantly better than placebo for reducing cough. Other drug combinations: EM-VIER was more effective than placebo for reducing cough in adults (reduced coughing fits $25 \% v 11 \%, \mathrm{p}<0.01$; reduced urge to cough $27 \% v 14 \%, \mathrm{p}<0.01)$. VICKS MEDINITE was rated as at least "good" for relieving cough by more adults in the treatment group than in the placebo group ( $58 \% v 32 \%$, $\mathrm{p}<0.01)$. A dextromethorphan/salbutamol combination improved night time cough compared with placebo or dextromethorphan alone (mean symptom score $0.19 v$ $0.67 v 0.44$ on $\mathrm{d} 4, \mathrm{p}<0.01)$. In children, 2 paediatric cough syrups (Triamincol syrup and Dorcol paediatric cough syrup) were not significantly different from placebo for parental report of satisfactory response. Antihistamines: 3 RCTs in adults showed that antihistamines (terfenadine or thonzylamine) were no better than placebo for resolving cough symptoms. 1 RCT in children showed that the antihistamines (clemastine and chlorpheniramine) were not better than placebo for improving cough scores.

\section{Conclusion}

In adults or children with acute cough, the evidence does not support the effectiveness of over the counter preparations.

\section{COMMENTARY}

In the first quantitative review of OTC cold medications, the only significant finding was that combination medications reduced cough in children $6-12$ years of age. ${ }^{1}$ The review by Schroeder and Fahey used a broad search strategy, and the selection of studies was not constrained by publication status or language, thus decreasing the risk of inclusion bias.

Schroeder and Fahey selected randomised, placebo controlled studies only and included adults and children. The studies on children were not stratified according to age group, yet physiological and anatomical differences occur across paediatric age groups. It is unclear whether the populations in the included studies were generalisable; for example, 1 study included children from private paediatric clinics.

One of the problems with the literature on OTC medications for cough is that the quality of studies varies, and many have serious methodological problems (in this review only 5 studies gave any description of the randomisation method). ${ }^{1}$

This review highlights the absence of good evidence, and we can conclude that more research is needed rather than that OTC cough remedies are ineffective. As a nurse practitioner, I advise patients against the purchase of cough remedies for acute non-productive cough because cough preparations have no proved efficacy; however, I might consider and suggest an antitussive if the cough interferes with sleep.

Johanne Mousseau, RN(EC), MSc Assistant Professor, McMaster University, School of Nursing Hamilton, Ontario, Canada

Smith MB, Feldman W. Over-the-counter cold medications: a critical review of clinical trials between 1950 and 1991. JAMA 1993;269:2258-63.

2 Luks D, Anderson M. Over-the-counter cold remedies. JAMA 1993;270:1812-3. 